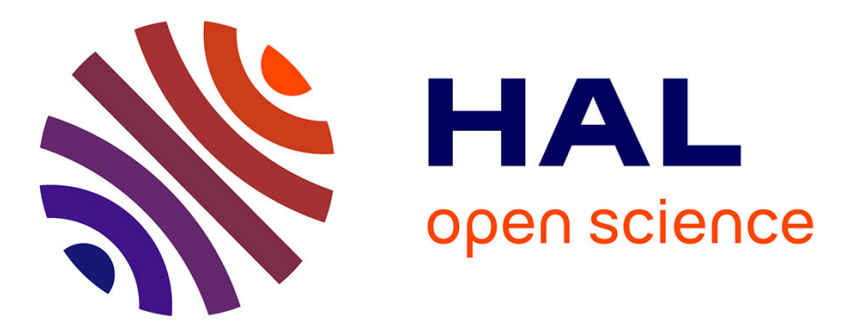

\title{
MC-CDMA System Identification and Equalization Using the LMS Algorithm and Takagi-Sugeno Fuzzy System
}

Said Safi, Miloud Frikel, Mathieu Pouliquen

\section{- To cite this version:}

Said Safi, Miloud Frikel, Mathieu Pouliquen. MC-CDMA System Identification and Equalization Using the LMS Algorithm and Takagi-Sugeno Fuzzy System. 11th IFAC International Workshop on Adaptation and Learning in Control and Signal Processing (ALCOSP'2013), Jul 2013, Caen, France. hal-01057707

\section{HAL Id: hal-01057707 https://hal.science/hal-01057707}

Submitted on 25 Aug 2014

HAL is a multi-disciplinary open access archive for the deposit and dissemination of scientific research documents, whether they are published or not. The documents may come from teaching and research institutions in France or abroad, or from public or private research centers.
L'archive ouverte pluridisciplinaire HAL, est destinée au dépôt et à la diffusion de documents scientifiques de niveau recherche, publiés ou non, émanant des établissements d'enseignement et de recherche français ou étrangers, des laboratoires publics ou privés. 


\title{
MC-CDMA System Identification and Equalization Using the LMS Algorithm and Takagi-Sugeno Fuzzy System
}

\author{
S. Safi ${ }^{*}$ M. Frikel and M. Pouliquen** \\ I. Badi, Y. Khmou and M. Boutalline ${ }^{* * *}$ \\ * Department of Mathematics and Informatics, Sultan Moulay Slimane \\ University, Po. Box: 592,Beni Mellal Morocco; (e-mail: safi.said@ \\ gmail.com). \\ ** ENSICAEN School, GREYC Laboratory, 6, B. Marchal Juin, 14050 \\ Caen France (e-mail; miloud.frikel@ensicaen.fr) \\ *** Polydisciplinary Faculty , Sultan Moulay Slimane University, Beni \\ Mellal Morocco.
}

\begin{abstract}
In this paper we are focused on the Multi-Carrier Code Division Multiple Access (MC-CDMA) equalization problem. The equalization is performed using the Minimum Mean Square Error (MMSE) and Zero Forcing (ZF) equalizer based on the identified parameters representing the indoor scenario (European Telecommunications Standards Institute Broadband Radio Access Networks (ETSI BRAN A) channel model), and outdoor scenario (ETSI BRAN E channel model). These channels are normalized for fourth-generation mobile communication systems. However, for such high-speed data transmissions, the channel is severely frequencyselective due to the presence of many interfering paths with different time delays. The identification problem is performed using the Least Mean Squares (LMS) algorithm and the Takagi-Sugueno (TS) fuzzy system. The comparison between these techniques, for the channel identification, will be made for different Signal to Noise Ratios (SNR).
\end{abstract}

Keywords: LMS algorithm; Takagi-Sugeno fuzzy system; MC-CDMA system; Identification and Equalization.

\section{INTRODUCTION}

Wireless or cellular mobile communication systems have been evolving according to advancements in wireless technologies and changes in user demands. In fixed and cellular networks, voice conversation was the dominant service for a long time. In line with the recent explosive expansion of Internet traffic in fixed networks, demands for broad ranges of services are becoming stronger even in mobile communication networks. A variety of services are now available over the second generation $(2 \mathrm{G})$ mobile communications systems, including email, Web access, and online services ranging from bank transactions to entertainment, in addition to voice conversation. 3G systems based on wide band direct sequence code-division multiple access (DSCDMA) Fazel et al [2008], with much higher data rates of up to $384 \mathrm{~kb} / \mathrm{s}$ (around $10 \mathrm{Mb} / \mathrm{s}$ in the later stage), were put into service in some countries, and their deployment speed has since accelerated. However, the capabilities of $3 \mathrm{G}$ systems will sooner or later be insufficient to cope with the increasing demands for broadband services that will soon be in full force in fixed networks. Demands for downloading of ever increasing volumes of information will become higher and higher. 4G systems that support extremely high-speed packet services are now expected to emerge Farhang: [1999], Safi et al [2009]. In this paper we focus on the channel identification representing the indoor propagation (ETSI BRAN A) and the outdoor propaga- tion (BRAN E), these channel models are normalized for $4 \mathrm{G}$ systems. the propagation channel is introduced for better understanding of the frequency-selective channel. The identification problem is performed using the Least Mean Squares algorithm (LMS) Farhang: [1999] and the Takagi-Sugueno (TS) fuzzy system Tanaka et al [2001].

\section{MC-CDMA (BROADBAND) CHANNEL}

Between a base station and a mobile station (MS) there are many obstacles, and also many local scatterers (e.g., neighboring buildings) in the vicinity of the MS. For this, the channel can be viewed as a time varying linear filter of impulse response $h(\tau)$ observed at time t, which can be expressed as Safi et al [2010]

$$
h(\tau)=\sum_{i=0}^{L-1} \xi_{i} \delta\left(\tau-\tau_{i}\right)
$$

where $\delta(n)$ is Dirac function, $\xi_{i}$ is the magnitude of the target $i, L=18$ the number of target and $\tau_{i}$ is the time delay (from the origin) of target $i$.

\subsection{ETSI BRAN A Mobile Channel Model}

In this paragraph we consider the ETSI BRAN A model representing the propagation in an indoor case. In the Ta- 
ble 1 we have summarized the measured values corresponding the ETSI BRAN A radio channel impulse response Eq. 1 .

Table 1. Delay and magnitudes of 18 targets of BRAN A radio channel

\begin{tabular}{|c|c||c|c|}
\hline \hline delay $\tau_{i}(n s)$ & mag. $C_{i}(d B)$ & delay $\tau_{i}(n s)$ & mag. $C_{i}(d B)$ \\
\hline 0 & 0 & 90 & -7.8 \\
10 & -0.9 & 110 & -4.7 \\
20 & -1.7 & 140 & -7.3 \\
30 & -2.6 & 170 & -9.9 \\
40 & -3.5 & 200 & -12.5 \\
50 & -4.3 & 240 & -13.7 \\
60 & -5.2 & 290 & -18 \\
70 & -6.1 & 340 & -22.4 \\
80 & -6.9 & 390 & -26.7 \\
\hline \hline
\end{tabular}

\subsection{ETSI BRAN E Mobile Channel Model}

The ETSI BRAN E model representing the fading radio channel, where the data corresponding to this model are measured in outdoor environment, Table 2.

Table 2. Delay and magnitudes of 18 targets of BRAN E channel

\begin{tabular}{|c|c||c|c|}
\hline \hline delay $\tau_{i}(n s)$ & mag. $C_{i}(d B)$ & delay $\tau_{i}(n s)$ & mag. $C_{i}(d B)$ \\
\hline 0 & -4.9 & 320 & 0 \\
10 & -5.1 & 430 & -1.9 \\
20 & -5.2 & 560 & -2.8 \\
40 & -0.8 & 710 & -5.4 \\
70 & -1.3 & 880 & -7.3 \\
100 & -1.9 & 1070 & -10.6 \\
140 & -0.3 & 1280 & -13.4 \\
190 & -1.2 & 1510 & -17.4 \\
240 & -2.1 & 1760 & -20.9 \\
\hline \hline
\end{tabular}

\section{APPLICATION: MC-CDMA SYSTEM}

The principles of MC-CDMA Linnartz [2001] is that a single data symbol is transmitted at multiple narrow band sub-carriers. Indeed, in MC-CDMA systems, spreading codes are applied in the frequency domain and transmitted over independent sub-carriers. However, multicarrier systems are very sensitive to synchronization errors such as symbol timing error, carrier frequency offset and phase noise. In this part, we describe a blind equalization techniques for MC-CDMA systems using the presented algorithm.

\subsection{MC-CDMA Transmitter}

The MC-CDMA modulator spreads the data of each user in frequency domain. In addition, precisely, the complex symbol $g_{i}$ of each user $i$ is, first, multiply by each chips $c_{i, k}$ of spreading code, and then apply to the modulator of multi-carriers. Each sub-carrier transmits an element of information multiply by a code chip of that sub-carrier. If We consider that: the length $L_{c}$ of spreading code is equal to the number $N_{p}$ of sub-carriers. Then The MC-CDMA signal is:

$$
s(t)=\frac{a_{i}}{\sqrt{N_{p}}} \sum_{k=0}^{N_{p}-1} c_{i, k} e^{2 j f_{k} t}
$$

where $f_{k}=f_{0}+\frac{1}{T_{c}}$

We suppose that, the channel is time invariant and it's impulse response is characterized by: $P$ paths of magnitudes $\beta_{p}$ and phase $\theta_{p}$. So the impulse response is given by

$$
h(\tau)=\sum_{p=0}^{P-1} \beta_{p} e^{j \theta_{p}} \delta\left(\tau-\tau_{p}\right)
$$

The relationship between the emitted signal $s(t)$ and the received signal $r(t)$ is given by: $r(t)=h(t) * s(t)+n(t)$

$$
r(t)=\sum_{p=0}^{P-1} \beta_{p} e^{j \theta_{p}} s(t-\tau)+n(t)
$$

where $n(t)$ is an additive white gaussian noise.

\section{2 $M C$-CDMA Receiver}

In a system of $N_{u}$ users, the emitted signal in a channel is

$$
s(t)=\sum_{u=0}^{N_{u}-1} \sum_{k=0}^{N_{p}-1} g_{u} c_{u, k} e^{2 j f_{k} t}
$$

The received signal after the introduction of the channel is: $r(t)=h(t) * s(t)+n(t)$.

$$
r(t)=\frac{1}{\sqrt{N_{p}}} \sum_{p=0}^{P-1} \sum_{k=0}^{N_{p}-1} \beta_{p} e^{j \theta} g_{u} c_{u, k} e^{2 j \pi\left(f_{0}+k / T_{s}\right)\left(t-\tau_{p}\right)}+n(t)
$$

At the reception, we demodulate the signal according the $N_{p}$ sub-carriers, and then we multiply the received sequence by the code of the user. When there are $\mathrm{M}$ active users, the received signal is

$$
r(t)=\sum_{m=0}^{M-1} \sum_{i=0}^{N-1} h_{m, i} c_{m}[i] a_{m}[k] \cos \left(2 \pi f_{c} t+2 \pi i \frac{F}{T_{b}} t+\theta_{m, i}\right)+n(t)
$$

where the effects of the channel have been included in $h_{m, i}$ and $\theta_{m, i}$ and $n(t)$ is additive white Gaussian noise (AWGN). Applying the receiver model to the received signal given in equation (5) yields the following decision variable for the $k^{\text {th }}$ data symbol assuming the users are synchronized in time

$$
\begin{gathered}
v_{0}=\sum_{m=0}^{M-1} \sum_{i=0}^{N_{p}-1} h_{m, i} c_{m}[i] g_{0, i} a_{m}[k] \frac{2}{T_{b}} \\
\int_{k T_{b}}^{(k+1) T_{b}} \cos \left(2 \pi f_{c} t+2 \pi i \frac{F}{T_{b}} t+\theta_{m, i}\right) \\
\cos \left(2 \pi f_{c} t+2 \pi i \frac{F}{T_{b}} t+\widehat{\theta}_{m, i}\right) d t+\eta
\end{gathered}
$$

where $\widehat{\theta}_{0, i}$ denotes the receiver's estimation of the phase at the $i^{t h}$ subcarrier of the desired signal. In the case of an ideal channel : $h_{m, i}=1, \theta_{m, i}=0$

$$
v_{0}=a_{0}[k] \sum_{i=0}^{N_{p}-1} c_{0}[i] c_{0}[i] g_{0, i}+\sum_{m=0}^{M-1} \sum_{i=0}^{N_{p}-1} a_{m}[k] c_{m}[i] c_{0}[i]+\eta
$$




$$
=a_{0}[k] \sum_{i=0}^{N_{p}-1} g_{0, i}+\eta
$$

The object of the equalization is to extract $a_{0}[k]$.

\section{EQUALIZATION FOR MC-CDMA}

\subsection{Zero forcing (ZF) Equalization technique}

The goal of the equalization techniques should be to reduce the effect of the fading and the interference while not enhancing the effect of the noise on the decision of what data symbol was transmitted. The principle of the $\mathrm{ZF}$, is to completely cancel the distortions brought by the channel. The gain factor of the ZF equalizer,is given by the equation

$$
g_{k}=\frac{1}{\left|h_{k}\right|}
$$

if we suppose that the spreading code are orthogonal, i.e.

$$
\sum_{q=0}^{N_{u}-1} \sum_{k=0}^{N_{p}-1} c_{j, k} c_{q, k}=0 \quad \forall q \neq j
$$

this fact, the estimated received symbol, $\hat{d}_{j}$ of symbol $d_{j}$ of the user $j$ is described by

$$
\hat{d}_{j}=\sum_{k=0}^{N_{p}-1} c_{j, k}^{2} d_{j}+\sum_{k=0}^{N_{p}-1} c_{j, k} \frac{1}{h_{k}} n k
$$

thus, the performance obtained using this detection techniques are independent of the users number, in condition that the spreading codes are orthogonal. But, if the $h_{k}$ value is very weak, (great fading cases), the values $g_{k}$ increases and the noise will be amplified.

\subsection{Minimum Mean Square Error (MMSE) Equalization}

The MMSE techniques combine the minimization the multiple access interference and the maximization of signal to noise ratio. The MMSE techniques minimize the mean squares error for each sub-carrier $k$ between the transmitted signal $s_{k}$ and the output detection $g_{k} r_{k}$ :

$$
\begin{aligned}
\varepsilon\left[|\epsilon|^{2}\right] & =\varepsilon\left[\left|s_{k}-g_{k} r_{k}\right|^{2}\right] \\
& =\varepsilon\left[\left(s_{k}-g_{k} h_{k} s_{k}-g_{k} n_{k}\right)\left(s_{k}^{*}-g_{k}^{*} h_{k}^{*} s_{k}^{*} g_{k}^{*} n_{k}^{*}\right)\right.
\end{aligned}
$$

the minimisation the function $\varepsilon\left[|\epsilon|^{2}\right]$, give us the optimal equalizer coefficient, under the minimization of the mean square error criterion, of each sub-carrier as:

$$
g_{k}=\frac{h_{k}^{*}}{\left|h_{k}^{*}\right|^{2}+\frac{1}{\gamma_{k}}}
$$

where $\gamma_{k}=\frac{E\left[\left|s_{k} h_{k}\right|^{2}\right]}{E\left|n_{k}\right|^{2}}$.

If the values $h_{k}$ are small, the $S N R$ for each sub-carrier is minimal. So, the use of the MMSE criterion avoid the noise amplification. On the other hand, the greatest values of the $h_{k}$, and $g_{k}$ being inversely proportional, allows to restore orthogonality between users.

\section{CHANNEL IDENTIFICATION USING THE LMS ALGORITHM AND TS FUZZY SYSTEM}

\subsection{Description of the LMS Algorithm}

From the method of steepest descent, the weight vector equation is given by Aboulnasr et al [1997]

$$
w(n+1)=w(n)+\frac{1}{2} \mu\left[-\nabla\left(E\left\{e^{2}(n)\right\}\right)\right]
$$

Where $\mu$ is the step-size parameter and controls the convergence characteristics of the LMS algorithm; $e^{2}(n)$ is the mean square error between the beamformer output $y(n)$ and the reference signal which is given by

$$
e^{2}(n)=\left[d^{*}(n)-w^{h} x(n)\right]^{2}
$$

The gradient vector in the above weight update equation can be computed as

$$
\nabla_{w}\left(E\left\{e^{2}(n)\right\}\right)=-2 r+2 R w(n)
$$

The LMS algorithm on the other hand simplifies this by using the instantaneous values of covariance matrices $r$ and $R$ instead of their actual values i.e.

$$
\begin{aligned}
R(n) & =x(n) x^{h}(n) \\
r(n) & =d^{*}(n) x(n)
\end{aligned}
$$

Therefore the weight update can be given by

$$
w(n+1)=w(n)+\mu x(n) e^{*}(n)
$$

The LMS algorithm is initiated with an arbitrary value $w(0)$ for the weight vector at $n=0$. The successive corrections of the weight vector eventually leads to the minimum value of the mean squared error. Therefore the LMS algorithm can be summarized in the following equations

$$
\begin{array}{cl}
\text { Output, } & y(n)=w^{h}(n) x(n) \\
\text { Error, } & e(n)=d^{*}(n)-y(n) \\
\text { Weight, } & w(n+1)=w(n)-\mu x(x) e^{*}(n)
\end{array}
$$

The LMS algorithm initiated with some arbitrary value for the weight vector is seen to converge and stay stable for

$$
0<\mu<1 / \lambda_{\max }
$$

Where $\lambda_{\max }$ is the largest eigenvalue of the correlation matrix $R$. The convergence of the algorithm is inversely proportional to the eigenvalue spread of the correlation matrix $R$. When the eigenvalues of $R$ are widespread, convergence may be slow. The eigenvalue spread of the correlation matrix is estimated by computing the ratio of the largest eigenvalue to the smallest eigenvalue of the matrix. If $\mu$ is chosen to be very small then the algorithm converges very slowly.

\subsection{Description of the TS fuzzy model}

The fuzzy model proposed by Takagi et al [1985] is described by fuzzy IF-THEN rules which represents local input-output relations of a nonlinear system. The main feature of a Takagi-Sugeno fuzzy model is to express the 
local dynamics of each fuzzy implication (rule) by a linear system model. The overall fuzzy model of the system is achieved by fuzzy "blending" of the linear system models Yong et al [2001]. In this work, we use particular fuzzy models of Takagi-Sugeno approach that allow non-linear systems by a combination of several local linear models Haiping et al [2008], Iqdour et al [2006]. These models are written as follows

$$
\begin{array}{r}
R_{i}: \text { if } x_{t} \text { is } A_{i} \text { Then } \hat{y}_{t, i}=\alpha_{0 i}+x_{t}^{T} \alpha_{i} \\
\text { where } i=1,2, \ldots c \text { and } t=1,2, \ldots N
\end{array}
$$

$R_{i}(i=1,2, \ldots, c)$ indicates the $i^{t h}$ fuzzy rule, $x_{t}$ is the input variable $\left(x_{t} \in \mathbf{R}^{\mathbf{n}}\right), \hat{y}_{t, i}$ is the output of the rule i relative to input $x_{i}, A_{i}$ is the fuzzy set and $\alpha_{i}=\left(\alpha_{1}, \alpha_{2}, \ldots, \alpha_{n}\right)^{T}$. The output $\hat{y}_{t}$ relative to input $x_{t}$ after aggregating of $\mathrm{c}$ TS fuzzy rules, can be written as a weighted sum of the individual conclusions

$$
\hat{y}_{t}=\sum_{i=1}^{c} \pi_{i}\left(x_{t}\right) \hat{y}_{t, i}
$$

with

$$
\pi_{i}\left(x_{t}\right)=\frac{\mu_{A_{i}}\left(x_{t}\right)}{\sum_{j=1}^{c} \mu_{A_{j}}\left(x_{t}\right)}
$$

where $\mu_{A_{i}}$ is the membership function related to the fuzzy set $A_{i}$. The identification of the TS fuzzy systems requires two types of tuning:

- Structural tuning: concerns the determination of the number of rules $\mathrm{c}$ and the fuzzy sets to be used in the fuzzy system, for that we used the Gustafson-Kessel (GK) fuzzy clustering algorithm Iqdour et al [2006] with the following fuzzy validity criterion

$$
S(c)=\sum_{t=1}^{N} \sum_{i=1}^{c}\left(\mu_{k t}\right)^{m}\left(\left\|z_{t}-\nu_{i}\right\|^{2}\left\|\nu_{i}-\bar{z}\right\|^{2}\right)
$$

Where $z_{t}$ is the $t^{t h}$ data point, $\nu_{i}$ is the center of the $i^{t h}$ cluster and $\bar{z}$ is the average of data and $\mathrm{m}$ is the fuzzification exponent. For the functional Eq.24, the two terms inside the bracket represent the variance of data inside each clusters and the variance of the clusters themselves, respectively. So the optimum number of clusters is determined as a minimum of the fuzzy validity criterion $S(c)$ as $c$ increases.

- Parametric tuning: consists to identify the parameters of the TS fuzzy model: Generally two methods are used for the estimation of the linear parameters $\left(\alpha_{k}\right)$ Tanaka et al [2001]. The first one is the Weighted Least Squares (WLS) algorithm, called also the local method. The second one is the Global Least Square (GLS) algorithm called also the global method. The determination of the best strategy to apply is not clearly established.

\section{SIMULATION RESULTS}

In this section we show the performance results obtained by computer simulation for different $S N R$ and assuming that the input channel is driven by non Gaussian signal $x(n)$. The output channel $y(n)$ is corrupted by a gaussian noise $N(n)$.
ETSI BRAN A Identification using the LMS algorithm In Fig. 1 we represent the estimation of the ETSI BRAN A parameters using the LMS algorithm, for an $S N R$ varying between $0 d B$ and $40 d B$ the data length is 2048 and for 100 iterations. From the Fig. 1 we observe a very low

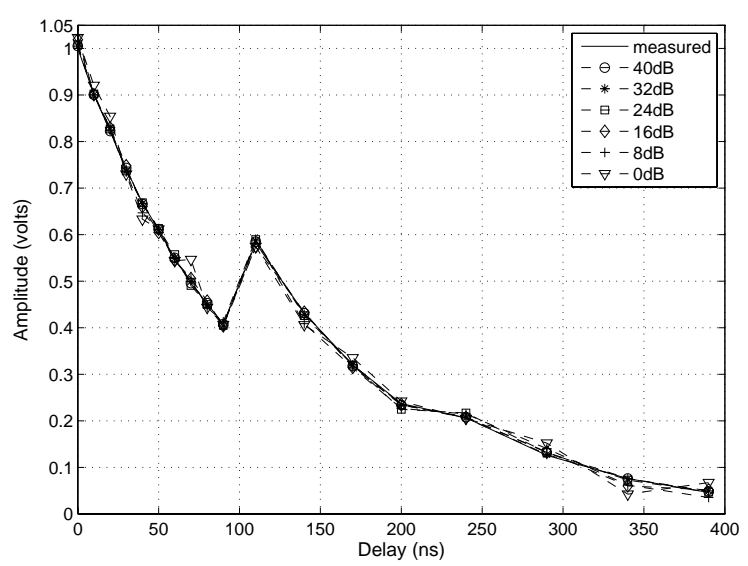

Fig. 1. ETSI BRAN A channel identification for different SNR, using the LMS algorithm

influence of the noise on the parameters estimation even for a $S N R=0 d B$, this is due to a slow variance of the impulse response of the ETSI BRAN A channel.

ETSI BRAN E Identification In this section we consider the ETSI BRAN E channel model. The Fig. 2 show the impulse response estimation for this channel using the LMS algorithm for different $S N R$. From the Fig. 2, we

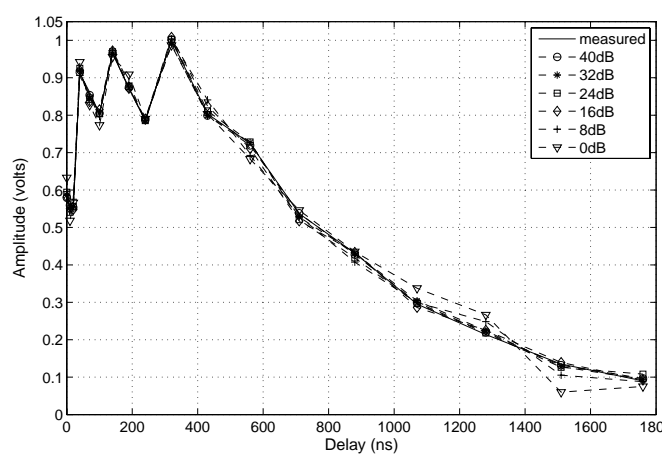

Fig. 2. ETSI BRAN E channel identification for different SNR, using the LMS algorithm

remark a slight influence of the noise in the impulse response parameters estimation principally if the $S N R<$ $24 d B$, but if the $S N R>24 d B$ the estimated parameters are very closed to the measured one.

\subsection{Channel Identification Using the TS Fuzzy System}

In this section we use Takagi-Sugueno fuzzy system to identify the impulse response of ETSI BRAN (A and E) channel model, for different $S N R$.

ETSI BRAN A Identification The Fig. 3 represent the impulse response of ETSI BRAN A channel model, for various $S N R$. 
This figure shows clearly the influence of noise on param-

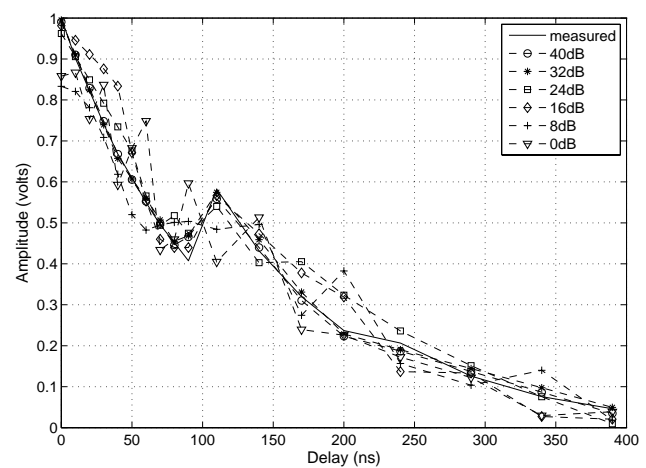

Fig. 3. ETSI BRAN A channel identification for different SNR, using TS fuzzy system

eter estimation, principally when the noise is important, i.e. $S N R<24 d B$, This is due to any noise filtering is realized in the TS fuzzy system. Which means if the noise is important, we observe that the estimated parameters are not closed to the measured one.

ETSI BRAN E Identification The problem of the noise filtering is most clear in the impulse response identification of the ETSI BRAN E channel model using the TS fuzzy system, i.e. Fig. 4, This figure demonstrates

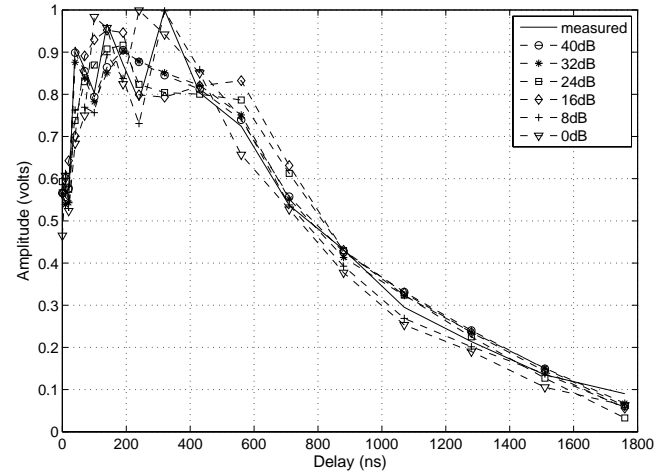

Fig. 4. ETSI BRAN E channel identification for different SNR, using TS fuzzy system

the identification problem of the ETSI BRAN E impulse response, when we have a rapid variance of the impulse response and in presence of noise, using the TS fuzzy system. This problem is clear principally for the first six values of the ETSI BRAN E impulse response, where the estimated parameters do not follow those measured. But, if the impulse response decrease "slowly", i.e after the sixth values we observe that the estimated values are closed to those measured.

\section{MC-CDMA SYSTEM PERFORMANCE}

In order to evaluate the performance of the MC-CDMA system, using the presented methods. These performances are evaluated by calculation of the Bit Error Rate (BER), for the two equalizers ZF and MMSE, using the measured and estimated (using the LMS algorithm and fuzzy system) BRAN A and BRAN E channel impulse response. The results are evaluated for different SNR values.

\subsection{ZF and MMSE equalizer: case of BRAN A channel}

In the Fig. 5, we represent the BER for different SNR, using the measured and estimated BRAN A channel using the LMS algorithm and fuzzy system. The equalization is performed using the ZF equalizer. From this figure we observe that: the LMS algorithm is more precise and gives good results than the TS fuzzy system.

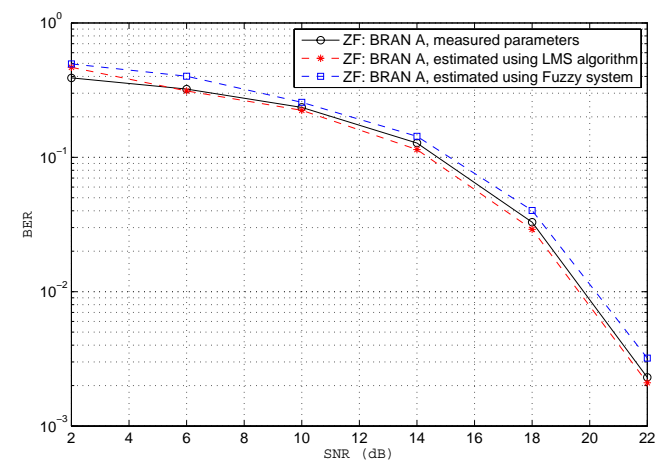

Fig. 5. BER of the estimated and measured BRAN A channel, for different SNR, using the ZF equalizer.

The Fig. 6 represents the BER for different SNR, using the measured and estimated BRAN A channel using the LMS algorithm and the Fuzzy system. The equalization is performed using the MMSE equalizer.

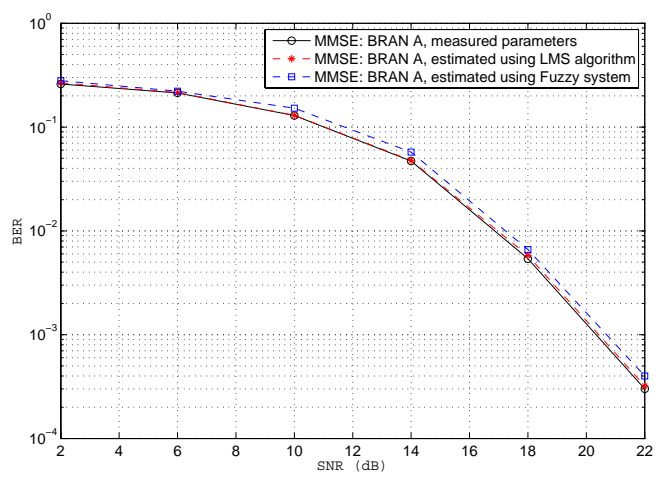

Fig. 6. BER of the estimated and measured BRAN A channel, for different SNR, using the MMSE equalizer.

The BER simulation for different $S N R$ demonstrates that the results obtained by the LMS algorithm have the same form comparing to the those obtained using the measured data. From the figures (Fig. 5 and 6 ) we conclude that: if the $S N R \geq 22 d B$ we have only a BER of $10^{-4}$. The BER values, obtained using the LMS algorithm, are more closed to those obtained using the measured values comparing to those obtained using the Fuzzy system, (Fig. 5 and 6).

\subsection{ZF and MMSE equalizer : case of BRAN E channel}

We represent in the Fig. 7, the simulation results of BER estimation using the measured and blind estimated of the BRAN E channel impulse response. The equalization is performed using ZF equalizer.

The Fig. 7 demonstrates clearly that the BER obtained, from ZF equalization, and based on the estimated parameters, using the LMS algorithm and Fuzzy system, ZF 


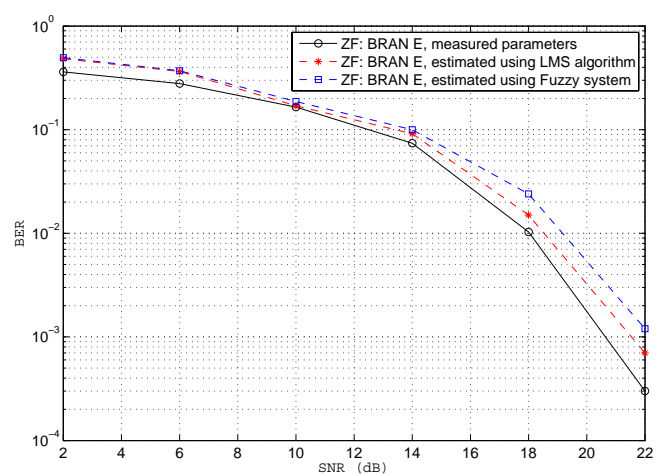

Fig. 7. BER of the estimated and measured BRAN E channel, for different SNR, using the ZF equalizer.

equalization gives good results like these obtained using measured values for ZF equalization. From Fig. 7 of the two techniques gives the 1 bit error if we receive $10^{4}$ if the $S N R \geq 22 d B$. In the same manner, we represent in Fig. 11 the simulation results of BER estimation using the measured and blind estimated of the BRAN E channel impulse response. The equalization is performed using MMSE equalizer.

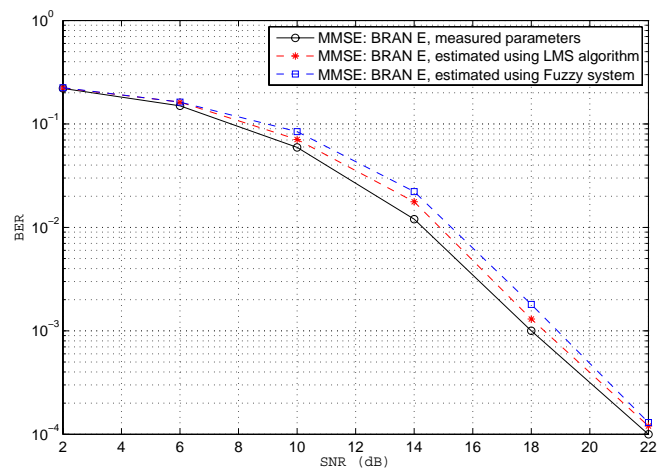

Fig. 8. BER of the estimated and measured BRAN E channel, for different SNR, using the MMSE equalizer.

From the Fig. 8, we observe that the blind MMSE equalization gives approximately the same results obtained using the measured BRAN E values for MMSE equalization. So, if the $S N R$ values are superior to $20 \mathrm{~dB}$, we observe that 1 bit error occurred when we receive $10^{3}$ bit, but if the $S N R \geq 22 d B$ we obtain only one bit error for $10^{4}$ bit received.

\section{CONCLUSION}

In this paper we have presented two techniques: LMS algorithm and TS fuzzy system. Both of those techniques are used to identify and equalize the MC-CDMA channel. The LMS algorithm show their efficiency in the impulse response channel (ETSI BRAN (A and E) normalized for the MC-CDMA system) identification with high precision (the estimated parameters are very closed to the measured one) for various $S N R$, eventually for a $S N R=0 d B$. The TS fuzzy system give good results for the impulse response ETSI BRAN A selective channel, for different $S N R>16 d B$. But for ETSI BRAN E selective channel we have some variance for the sixth values of the impulse response.

\section{REFERENCES}

K. Fazel and S.Kaiser. Multi-Carrier and Spread Spectrum Systems From OFDM and MC-CDMA to LTE and WiMAX. Second edition, John Wiley E Sons, Ltd. 2008.

B. Farhang-Boroujeny. Adaptive Filters Theory and Applications. New York: Wiley, 1999.

S. Safi, M. Fakir, M. Frikel and M. M'Saad. Blind identification of indoor broadband radio access network using cumulants. International Conference on Multimedia Computing and Systems, ICMCS '09, pages 249 - 252, 2009.

K. Tanaka, H. Ohtake and H.O. Wang. Model construction, rule reduction, and robust compensation for generalized form of Takagi-Sugeno fuzzy systems IEEE Transactions on Fuzzy Systems, Volume 9 , no. 4, pages 525-538, June 2001.

T. Aboulnasr and K. Mayyas. A robust variable step-size LMS-type algorithm: analysis and simulations IEEE Transactions on Signal Processing, Volume 45, no. 3, pages 631 - 639, 1997.

S. Safi, M. Frikel, B. Targui, E. Cherrier, M. Pouliquen and M. M'saad. Identification and equalization of MCCDMA system driven by stochastic chaotic code, $18^{\text {th }}$ Mediterranean Conference on Control \& Automation (MED), pp. 1643 - 1649, 2010.

A. Wee-Peng and B. Farhang-Boroujeny. A new class of gradient adaptive step-size LMS algorithms, IEEE Transactions on Signal Processing, Volume 49, no. 4, pages. $805-810,2001$.

T. Takagi and M. Sugeno. Fuzzy identification of systems and its applications to modeling and control. IEEE Trans Systems Man and Cybernetics, Volume 15, pages. 116 - 132, 1985.

Yong-Yan Cao and P.M. Frank. Stability analysis and synthesis of nonlinear time-delay systems via linear TakagiSugeno fuzzy models. Fuzzy Sets and Systems, Elsevier, Volume 124, no.2, pages 213-229, 2001.

Haiping Du and Nong Zhang. Application of evolving TakagiSugeno fuzzy model to nonlinear system identification. Applied Soft Computing, Elsevier, Vol. 8, no. 1, pages. 676-686, 2008.

R. Iqdour and A. Zeroual. A rule based fuzzy model for the prediction of solar radiation. Revue des Energies Renouvelables Volume 9, no.2, pages 113 - 120, 2006.

L. Zadeh. Fuzzy Sets. Information and Control, Volume 8, pages 338- 353, 1965.

A. Fiordaliso. Auto-Structuration of Fuzzy Systems y RulesSensitivity Analysis. Fuzzy Sets and Systems, Volume 118, pages 581 -586, 2001.

Jean-Paul M. G. Linnartz. Performance Analysis of Synchronous MC-CDMA in Mobile Rayleigh Channel With Both Delay and Doppler Spreads. em IEEE Transactions on Vehicular Technology, Volume 50, no. 6, pages, 1375-1387, November 2001.

S. Kaiser. OFDM-CDMA versus DS-CDMA: performance evaluation for fading channels. IEEE International Conference on Communications, pages, 1722-1726, Juin 1995. 